\title{
A gravitational lensing detection of filamentary structures connecting luminous red galaxies
}

\author{
Qianli Xia ${ }^{1}$, Naomi Robertson ${ }^{2}$, Catherine Heymans ${ }^{1,3}$, Alexandra Amon ${ }^{1,4}$, Marika Asgari ${ }^{1}$, Yan-Chuan Cai ${ }^{1}$, \\ Thomas Erben ${ }^{5}$, Joachim Harnois-Déraps ${ }^{1}$, Hendrik Hildebrandt ${ }^{3}$, Arun Kannawadi ${ }^{6}$, Konrad Kuijken ${ }^{6}$, \\ Peter Schneider ${ }^{5}$, Cristóbal Sifón ${ }^{7}$, Tilman Tröster ${ }^{1}$, and Angus H. Wright ${ }^{3}$ \\ ${ }^{1}$ Institute for Astronomy, University of Edinburgh, Royal Observatory, Blackford Hill, Edinburgh EH9 3HJ, UK \\ e-mail: qx211@roe.ac.uk \\ 2 Department of Astrophysics, University of Oxford, Keble Road, Oxford OX1 3RH, UK \\ e-mail: naomi .robertson@physics.ox.ac.uk \\ ${ }^{3}$ German Centre for Cosmological Lensing, Astronomisches Institut, Ruhr-Universität Bochum, Universitätsstr, Bochum, Germany \\ ${ }^{4}$ Kavli Institute for Particle Astrophysics \& Cosmology, Stanford University, PO Box 2450, Stanford, CA 94305, USA \\ 5 Argelander-Institut für Astronomie, Universitẗ Bonn, Auf dem Hügel 71, 53121 Bonn, Germany \\ ${ }^{6}$ Leiden Observatory, Leiden University, PO Box 9513, 2300 Leiden, The Netherlands \\ 7 Instituto de Física, Pontificia Universidad Católica de Valparaíso, Casilla 4059, Valparaíso, Chile
}

Received 12 September 2019 / Accepted 26 November 2019

\begin{abstract}
We present a weak lensing detection of filamentary structures in the cosmic web, combining data from the Kilo-Degree Survey, the Red Cluster Sequence Lensing Survey, and the Canada-France-Hawaii Telescope Lensing Survey. The line connecting luminous red galaxies with a separation of $3-5 h^{-1} \mathrm{Mpc}$ was chosen as a proxy for the location of filaments. We measured the average weak lensing shear around $\sim 11000$ candidate filaments selected in this way from the Sloan Digital Sky Survey. After nulling the shear induced by the dark matter haloes around each galaxy, we reported a $3.4 \sigma$ detection of an anisotropic shear signal from the matter that connects them. Adopting a filament density profile, motivated from $N$-body simulations, the average density at the centre of these filamentary structures was found to be $15 \pm 4$ times the critical density.
\end{abstract}

Key words. gravitational lensing: weak - large-scale structure of Universe - dark matter - cosmology: observations

\section{Introduction}

Galaxy surveys, including the 2dF Galaxy Redshift Survey (Colless et al. 2001) and the Sloan Digital Sky Survey (SDSS; Zehavi et al. 2011), have shown that visible matter in our Universe is not uniformly distributed on intermediate scales $\sim 100 \mathrm{~h}^{-1} \mathrm{Mpc}$. Instead, a web-like structure is observed with clusters of galaxies identifying the densest regions. $N$-body simulations predict the existence of these large-scale structures (e.g. Bond et al. 1996; Springel et al. 2005), suggesting a hierarchical structure formation for the cosmic web. We can classify the web (e.g. Eardley et al. 2015) into regions of clusters, filaments, sheets and voids. In this cosmic web, large under-dense regions (voids) are enclosed by anisotropically collapsed surface structures (sheets) and line structures (filaments) which intersect at the most over-dense isotropic regions (clusters). The Zel'dovich approximation predicts that $\sim 42 \%$ of the mass of the Universe is in a filament environment (Zel'dovich 1970), and this has been confirmed by simulations (Aragón-Calvo et al. 2010). However, as filament environments do not display a very high density contrast, this makes direct observations challenging.

One way to observe filaments is from the X-ray emission induced by the warm hot intergalactic medium (WHIM) with several inter-cluster filaments investigated in this way (Briel \& Henry 1995; Kull \& Böhringer 1999; Werner et al. 2008). There are also reported detections of filaments using overdensities of galaxies (Pimbblet \& Drinkwater 2004; Ebeling et al.
2004). Recently, two independent studies (de Graaff et al. 2019; Tanimura et al. 2019) detected the Sunyaev-Zel'dovich (SZ) signal from the ionised gas in the cosmic web. They estimated the density of ionised gas to be $\sim(28 \pm 12) \%$ of the total baryon density in the Universe, close to resolving the missing baryon problem (Bregman 2007).

In this paper, we investigate the use of weak gravitational lensing to detect filaments. Based on the distortion of light rays around massive objects, gravitational lensing probes the total mass traced by the large-scale structures and is, therefore, highly complementary to the SZ detection of the gas. Although Dietrich et al. (2012) made a direct weak lensing detection of a filament connecting two massive Abell clusters, the direct detection of typical individual filaments is limited by the low signalto-noise measurement, and most studies stack large samples of candidate filaments and analyse the resulting average weak lensing signal instead (Mead et al. 2010; Clampitt et al. 2016; Epps \& Hudson 2017; Kondo et al. 2019).

Clampitt et al. (2016; hereafter C16) determined the weak lensing signal at around 135000 pairs of SDSS Luminous Red Galaxies (LRGs) with a projected separation $6 h^{-1} \mathrm{Mpc} \leq$ $R_{\text {sep }} \leq 14 h^{-1} \mathrm{Mpc}$ and a redshift separation of $\Delta z<0.004$. Using a "nulling" estimator that cancels the spherically symmetric contribution of the LRG haloes in the shear measurement, they reported a $4.5 \sigma$ detection of the filament lensing signal. In another study, Epps \& Hudson (2017) used 23000 
pairs of LRGs from the Baryon Oscillation Spectroscopic Survey (BOSS) LOWZ and CMASS samples as tracers of filaments. Using data from the Canada-France-Hawaii Telescope Lensing Survey (CFHTLenS), they performed a mass reconstruction of a set of stacked LRG pairs with a projected angular separation between 6 and $10 h^{-1} \mathrm{Mpc}$ and a redshift separation $\Delta z<0.003$. After subtracting the signal from a mass reconstruction of a set of stacked LRG pairs with the same separation on the sky but a greater redshift separation $(0.033<\Delta z<0.04)$ such that haloes should not be physically connected, they reported a $5 \sigma$ detection of a filament lensing signal. A more recent study (Kondo et al. 2019) used 70210 pairs of LRGs from the CMASS sample with a projected separation between 6 and $14 h^{-1} \mathrm{Mpc}$ and a line-of-sight separation of less than $6 h^{-1} \mathrm{Mpc}$. Using the Subaru Hyper Suprime-Cam (HSC) first-year galaxy shape catalogue and adopting the $\mathrm{C} 16$ nulling approach they reported $3.9 \sigma$ detection of a filament signal.

We note that the methodology taken in these previous studies can be understood as a three-point galaxy-galaxy-shear correlation function conditioned on specific intervals of separation between lens galaxies (Schneider \& Watts 2005). The full suite of the galaxy-galaxy-galaxy lensing (GGGL) statistics have been applied to the Red-Sequence Cluster Survey (Simon et al. 2008) and CFHTLenS (Simon et al. 2019) to measure the excess mass around galaxy pairs separated by $\lesssim 300 h^{-1} \mathrm{kpc}$.

In this paper, we present the weak lensing signal measured between 11706 LOWZ LRG pairs that have a separation of $3-5 h^{-1} \mathrm{Mpc}$, combining three public weak lensing surveys; the KiDS+VIKING-450 survey (KV450; Hildebrandt et al. 2018; Wright et al. 2019), the Red Cluster Sequence Lensing Survey (RCSLenS; Hildebrandt et al. 2016), and the CFHTLenS (Heymans et al. 2012). We improve the nulling methodology described in $\mathrm{C} 16$ to deal with contamination from filament tracers and use a large suite of $N$-body simulations to validate our pipeline and compare our results. A standard $\Lambda$ CDM cosmology has been adopted throughout this study to calculate distances with a matter density $\Omega_{\mathrm{m}}=0.3$, energy density $\Omega_{\Lambda}=0.7$, effective number of neutrino species $N_{\text {eff }}=3.04$, baryon density $\Omega_{\mathrm{b}}=0.0$ and current Hubble constant $H_{0}=100 h \mathrm{~km} \mathrm{~s}^{-1} \mathrm{Mpc}^{-1}$ where $h$ is the Hubble parameter $h=0.7$.

This paper is structured as follows. In Sect. 2, we describe the survey data and simulations. Section 3 summarises the weak lensing formalism, the adopted filament model, and methodology. We show our results in Sect. 4 and conclude in Sect. 5. In Appendix A, we present a validation of our nulling technique. In Appendix B, we document the spherical rotation methodology that is required for high declination surveys.

\section{Surveys and simulations}

\subsection{The lensing surveys}

The properties of the three lensing surveys, KV450, RCSLenS, and CFHTLenS, are listed in Table 1 . They share a similar data processing pipeline, where the shape measurement of galaxies was conducted using the lensfit model fitting code (Miller et al. 2013). This approach convolves the pixelised model PointSpread-Function with an analytical surface brightness model consisting of bulge and disk components. It uses model fitting to estimate galaxy ellipticitices $\epsilon_{1}^{\mathrm{obs}}$ and $\epsilon_{2}^{\mathrm{obs}}$ with an associated inverse variance weight, $w_{\mathrm{s}}$. The (reduced) shear (cf. Eq. (4)) is then given by the weighted average of ellipticities, $\gamma_{i}^{\text {obs }} \approx$ $\sum_{\mathrm{s}} w_{\mathrm{s}} \epsilon_{i}^{\mathrm{obs}} / \sum_{\mathrm{s}} w_{\mathrm{s}}(i=1,2)$. The observed shear is biased with respect to the true shear and is typically described by the linear bias model (Heymans et al. 2006) as

$\gamma^{\mathrm{obs}}=(1+m) \gamma^{\text {true }}+c$,

In all of these three surveys, the shear multiplicative bias terms were characterised as a function of the signal-to-noise ratio and size of the galaxies, thereby allowing us to calculate the bias for an arbitrary selection of galaxies. The correction for this multiplicative bias was carried out as it was in Velander et al. (2014).

Photometric redshifts, $z_{\mathrm{B}}$, were estimated using the Bayesian photometric redshift algorithm (BPZ; Benítez 2000) as detailed in Hildebrandt et al. (2012). Wright et al. (2019) and Hildebrandt et al. (2018) show how the photometric redshifts distributions for KV450 are then calibrated using the "weighted direct calibration" method, with weights estimated using a deep spectroscopic training sample in 9-band ugriZY JHK magnitude space. No such calibration was performed for the 4-band (RCSLenS) or 5-band (CFHTLenS) surveys, and instead a probability distribution of true redshifts was estimated from the sum of the BPZ redshift probability distributions. This approach has been demonstrated to carry more systematic error (Choi et al. 2016; Hildebrandt et al. 2017). We discuss how we take this redshift uncertainty into account in our final analysis in Sect. 3.4.

\subsection{The BOSS survey}

We used the Baryon Oscillation Spectroscopic Survey (BOSS) galaxies from the 12th SDSS Data Release (Alam et al. 2015) to define candidate filaments. Among all three lensing surveys RCSLenS has the most SDSS overlap with almost double that of KV450 or CFHTLenS. Once a robust photometric redshift selection has been applied, however, RCSLenS has only 20\%/30\% the lensing source density in comparison to CFHTLenS/KV450, (see Table 1 for details).

Given the depth of the lensing surveys and the uncertainty in the high redshift tail of the redshift distribution for CFHTLenS and RCSLenS, we chose to limit our analysis to the LOWZ sample, selected based on colour and magnitude, using a redshift cut $0.15<z<0.43$ (Ross et al. 2012). We did not consider the higher redshift CMASS sample. The typical virial halo mass of LOWZ galaxies is $\sim 5.2 \times 10^{13} h^{-1} M_{\odot}$ (Parejko et al. 2013). These haloes have a typical virial radius $\sim 1 h^{-1} \mathrm{Mpc}$.

\subsection{Simulations}

We used the Scinet Light Cone Simulations (SLICS ${ }^{1}$; Harnois-Déraps \& van Waerbeke 2015; Harnois-Déraps et al. 2018 ) to test our methodology. This suite provided us with 819 independent light cones on a $10 \times 10 \mathrm{deg}^{2}$ patch of the sky. Each light cone was constructed from the full non-linear evolution of $1536^{3}$ particles with $m_{\mathrm{p}}=2.88 \times 10^{9} h^{-1} M_{\odot}$, within a $505^{3}\left(h^{-1} \mathrm{Mpc}\right)^{3}$ cube. Particles were then projected onto mass sheets at 18 redshifts between $0<z<3$, and subsequently inspected to identify dark matter haloes. For each simulation, $100 \mathrm{deg}^{2}$ light cone mass sheets and haloes were extracted; the former were then ray-traced into lensing shear maps, while the latter were used to generate mock LOWZ galaxies with a halo occupation distribution, optimised so that the clustering of mock galaxies would be consistent with the LOWZ data (see Harnois-Déraps et al. 2018 for details). We find the mock sample variance to be in good agreement with the Jack-knife errors measured directly from the data. Source galaxy positions were

\footnotetext{
http://slics.roe.ac.uk
} 
Table 1. Properties of each of the three lensing surveys used in this analysis.

\begin{tabular}{lccc}
\hline \hline & KV450 & RCSLenS & CFHTLenS \\
\hline Total area $\left(\mathrm{deg}^{2}\right)$ & 454 & 785 & 154 \\
Unmasked area $\left(\mathrm{deg}^{2}\right)$ & 341.3 & 571.7 & 146.5 \\
Total LOWZ overlap area $\left(\mathrm{deg}^{2}\right)$ & 135.91 & 224.63 & 113.83 \\
$z_{\mathrm{B}}$ selection & $0.1<z_{\mathrm{B}}<1.2$ & $0.4<z_{\mathrm{B}}<1.1$ & $0.2<z_{\mathrm{B}}<1.3$ \\
$n_{\text {eff }}(\operatorname{arcmin}$ & \\
Photometric bands & 6.93 & 2.2 & 11 \\
\hline
\end{tabular}

Notes. The table lists: the total and effective unmasked survey area, the total LOWZ overlap area, photometric redshfit, $z_{\mathrm{B}}$, selection and the effective number density of lensing sources $n_{\mathrm{eff}}$ under the corresponding $z_{\mathrm{B}}$ selection. For the $z_{\mathrm{B}}$ selection, we followed Hildebrandt et al. (2017), Hildebrandt et al. (2016), and Heymans et al. (2012), respectively.

drawn at random, with the shear assigned for a range of redshifts and high number density. We next randomly drew from the SLICS mock source galaxy sample so as to match the corresponding number density and redshift distribution of each of the three surveys, KV450, RCSLenS, and CFHTLenS. Intrinsic galaxy shapes were chosen to match the KiDS ellipticity dispersion (Hildebrandt et al. 2017), which is good description of the ellipticity dispersion that is also found in RCSLenS and CFHTLenS.

\section{Summary of weak lensing formalism and methodology}

In this section, we summarise the weak gravitational lensing theory following the more detailed derivations in Bartelmann \& Schneider (2001). Assuming the thin lens approximation, a foreground object at a position $\boldsymbol{\theta}$ has a $2 \mathrm{D}$ comoving surface mass density $\Sigma(\boldsymbol{\theta})$. The convergence is then defined as

$\kappa(\boldsymbol{\theta})=\frac{\Sigma(\boldsymbol{\theta})}{\Sigma_{\text {crit }}}$

where $\Sigma_{\text {crit }}$ is the comoving critical surface density in a flat Universe given by

$\Sigma_{\text {crit }}=\frac{c^{2}}{4 \pi G} \frac{\chi\left(z_{\mathrm{s}}\right)}{\left[\chi\left(z_{\mathrm{s}}\right)-\chi\left(z_{1}\right)\right] \chi\left(z_{1}\right)\left(1+z_{1}\right)}$.

Here $\chi$ is the comoving distance and $z_{1}, z_{\mathrm{s}}$ are the redshifts of the lens and source, respectively. Since we are interested in the large scale comoving surface density around filaments, this is the appropriate choice for $\Sigma_{\text {crit }}$ (see Dvornik et al. 2018, for a discussion on the different definitions of $\Sigma_{\text {crit }}$ ).

The deflection potential, $\psi(\theta)$, is connected to the convergence, $\kappa$, via Poisson's equation $\nabla^{2} \psi=2 \kappa$, and the complex shear is related to the second derivatives of the deflection potential via

$\gamma=\gamma_{1}+i \gamma_{2}=\frac{1}{2}\left(\frac{\partial^{2} \psi}{\partial x_{1}^{2}}-\frac{\partial^{2} \psi}{\partial x_{2}^{2}}\right)+i \frac{\partial^{2} \psi}{\partial x_{1} \partial x_{2}}$,

where $x_{1}, x_{2}$ are the horizontal and vertical displacements on the projected sky.

For a filament aligned with the $x_{1}$ axis if we assume the deflection potential $\psi$ and convergence $\kappa$ are both invariant along the filament, then Eq. (4) immediately implies that partial derivatives with respect to the $x_{1}$-axis will be equal to zero. This leads to the approximation that for filaments, we should expect to measure $\gamma_{1} \approx-\kappa$ and $\gamma_{2} \approx 0$. Motivated by the simulation results of Colberg et al. (2005) and Mead et al. (2010) considered the power law density profile around filaments and suggested the model for the convergence at a distance $r$ from filament centre, measured perpendicular to the major filament axis ( $x_{1}$-axis)

$\kappa(r) \approx \frac{\kappa_{\mathrm{c}}}{1+\left(\frac{r}{r_{\mathrm{c}}}\right)^{2}}$.

Here $\kappa_{\mathrm{c}}$ is the amplitude of the convergence at the filament centre $(r=0)$ and $r_{\mathrm{c}}$ is the half-maximum radius of the density profile.

\subsection{Filament candidiate}

Colberg et al. (2005) showed that cluster pairs separated by $<5 h^{-1} \mathrm{Mpc}$ are always connected by filamentary structures. We therefore select luminous red galaxy (LRG) pairs in the LOWZ catalogue with redshift separation $\delta z<0.002$ and a projected separation $3 h^{-1} \mathrm{Mpc} \leq R_{\text {sep }} \leq 5 h^{-1} \mathrm{Mpc}$ as our candidate filaments ${ }^{2}$ which we will refer to as our physical pairs (PP). Non-physical pairs (NP) are defined to have the same projected separation range but with large line-of-sight separations with $0.033<\delta z<0.04$ (corresponding to $\sim 100 \mathrm{~h}^{-1} \mathrm{Mpc}$ ). With such a large physical separation we would not expect to detect a filament signal. The NP therefore provide an important null-test for our methodology.

Our candidates differ from the selection made by $\mathrm{C} 16$, Epps \& Hudson (2017), de Graaff et al. (2019), Tanimura et al. (2019), and Kondo et al. (2019), who focused on separations of 6-10 $h^{-1} \mathrm{Mpc}$. Our choice maximises signal-to-noise, as shown in our analysis of numerical simulations in Sect. 4.1, but for completeness we also present an analysis of $6-10 h^{-1} \mathrm{Mpc}$ filaments in Sect. 5.

\subsection{Stacking method}

For each lens filament candidate at redshift $z_{1}=z_{\mathrm{f}}=\left(z_{\text {lens } 1}+\right.$ $z_{\text {lens } 2)} / 2$, we measure the $\Sigma_{\text {crit }}$-weighted shear, $E_{\mathrm{f}}$, on a grid $(i, j)$ centred and oriented with the pair of LRGs, where

$E_{\mathrm{f}}(i, j)=\sum_{\mathrm{s}} w_{\mathrm{s}} \overline{\Sigma_{\text {crit }}^{-1}}\left(z_{\mathrm{l}}\right) \widetilde{\epsilon}_{\mathrm{s}}^{\mathrm{obs}} \Theta_{\mathrm{s}}(i, j)$,

and the sum is taken over all sources ${ }^{3}, s$, with $z_{\mathrm{B}}>z_{1}+0.1$ and

$\Theta_{\mathrm{s}}(i, j)= \begin{cases}1 & \text { if source, } s, \text { lies in pixel }(i, j), \\ 0 & \text { otherwise. }\end{cases}$

2 The average 3D separation between these filament candidates is about $7 h^{-1}$ Mpc.

3 We use a redshift cut $z_{\mathrm{B}}-z_{1}>0.1$ everywhere to ensure that the majority of our source galaxies are behind the foreground galaxies, and not associated with them. Fig. D3 in Amon et al. (2018) demonstrates that with this selection, contamination of the KiDS source sample is negligible for the scales we probe at $>0.1 h^{-1} \mathrm{Mpc}$. 
The complex ellipticity $\widetilde{\epsilon}_{\mathrm{s}}^{\mathrm{obs}}=\widetilde{\epsilon}_{1}^{\mathrm{obs}}+\widetilde{\epsilon}_{2}^{\mathrm{obs}}$ is the observed ellipticity of the source rotated into the reference frame where the filament lies along the $x_{1}$ axis. The $\Sigma_{\text {crit }}$ weight converts from a shear estimate to an estimate of the surface mass density $\Sigma$ in Eq. (2). The grid $(i, j)$ has an extent $\left[-2 R_{\text {sep }}, 2 R_{\text {sep }}\right] \times\left[-2 R_{\text {sep }}, 2 R_{\text {sep }}\right]$ and $129^{2}$ pixels, and the pair of lens galaxies that define the filament candidate are positioned to lie at the centre of the pixels at $\left(-0.5 R_{\text {sep }}, 0\right)$ and $\left(0.5 R_{\text {sep }}, 0\right)$.

We also construct a corresponding weight map for each filament candidate,

$W_{\mathrm{f}}(i, j)=\sum_{\mathrm{s}} w_{\mathrm{s}}\left[\overline{\Sigma_{\text {crit }}^{-1}}\left(z_{\mathrm{l}}\right)\right]^{2} \Theta_{\mathrm{s}}(i, j)$,

where the extra factor of $\overline{\Sigma_{\text {crit }}^{-1}}\left(z_{1}\right)$ provides optimal signal-tonoise weighting (Velander et al. 2011).

When rotating each filament pair into a common reference frame, we note that at high declination, the tangent plane method used in Epps \& Hudson (2017) and the direct cartesian approximation in $\mathrm{C} 16$ results in non-uniform grid cells. As the nulling approach requires a flat geometry on the grid, we found that these approximations lead to a biased result. To solve this problem for high-declination patches, we use the spherical rotation method from de Graaff et al. (2019). This process is detailed in Appendix B, and illustrated in Fig. B.1, with the rotated shear map $\left(\widetilde{\epsilon_{1}}, \widetilde{\epsilon}_{2}\right)$ defined in Eq. (B.4).

As we have spectroscopic redshifts for the filaments but only photometric redshifts for the sources, the inverse critical surface mass density $\Sigma_{\text {crit }}^{-1}\left(z_{1}\right)$ is calculated for each survey as

$$
\begin{aligned}
\overline{\Sigma_{\text {crit }}^{-1}}\left(z_{\mathrm{l}}\right) & \equiv \int_{z_{\mathrm{l}}}^{\infty} \mathrm{d} z_{\mathrm{s}} \overline{p_{\mathrm{s}}}\left(z_{\mathrm{s}}, z_{\mathrm{l}}\right) \Sigma_{\text {crit }}^{-1}\left(z_{\mathrm{l}}, z_{\mathrm{s}}\right) \\
& =\frac{4 \pi G\left(1+z_{\mathrm{l}}\right) \chi\left(z_{\mathrm{l}}\right)}{c^{2}} \int_{z_{\mathrm{l}}}^{\infty} \mathrm{d} z_{\mathrm{s}} \overline{p_{\mathrm{s}}}\left(z_{\mathrm{s}}, z_{\mathrm{l}}\right)\left[1-\frac{\chi\left(z_{\mathrm{l}}\right)}{\chi\left(z_{\mathrm{s}}\right)}\right],
\end{aligned}
$$

where $\overline{p_{\mathrm{s}}}$ is the probability distribution of the true redshift of the source galaxies that enter the measurement

$\overline{p_{\mathrm{s}}}\left(z_{\mathrm{s}}, z_{\mathrm{l}}\right)=\frac{\sum_{s} w_{\mathrm{s}} p_{\mathrm{s}}\left(z_{\mathrm{s}} \mid z_{\mathrm{B}}\right)}{\sum_{s} w_{\mathrm{s}}}$.

For CFHTLenS and RCSLenS we use the per-source $p_{\mathrm{s}}\left(z_{\mathrm{s}} \mid z_{\mathrm{B}}\right)$ provided by each survey, even though this has been shown to introduce biases (Choi et al. 2016), which we account for in Sect. 3.4. For KV450 we use the weighted direct calibration method of Hildebrandt et al. (2017) to determine the source redshift distribution $\overline{p_{\mathrm{s}}}\left(z_{\mathrm{s}}\right)$ directly for an ensemble of sources. In practice we calculate $\overline{\Sigma_{\text {crit }}^{-1}}$ in Eq. (10) at eight $z_{1}$ values and interpolate to evaluate $\overline{\Sigma_{\text {crit }}^{-1}}$ at each filament redshift. The effective $n\left(z_{\mathrm{s}}\right)$ for each survey, given by

$n\left(z_{\mathrm{s}}\right)=\int \overline{p_{\mathrm{s}}}\left(z_{\mathrm{s}}, z_{\mathrm{l}}\right) p\left(z_{\mathrm{l}}\right) \mathrm{d} z_{\mathrm{l}}$,

is shown in Fig. 1, with CFHTLenS and KV450 providing a deeper source redshift than RCSLenS.

We correct the measured shear signal $E_{\mathrm{f}}$, with the signal measured around "random" filaments. This is now a standard procedure in galaxy-galaxy lensing studies (e.g. Mandelbaum et al. 2005) which removes any hidden systematics and reduces sampling variance noise. We create random filament samples for each survey patch, listed in Table 2, by randomly shifting filaments within the same patch while preserving

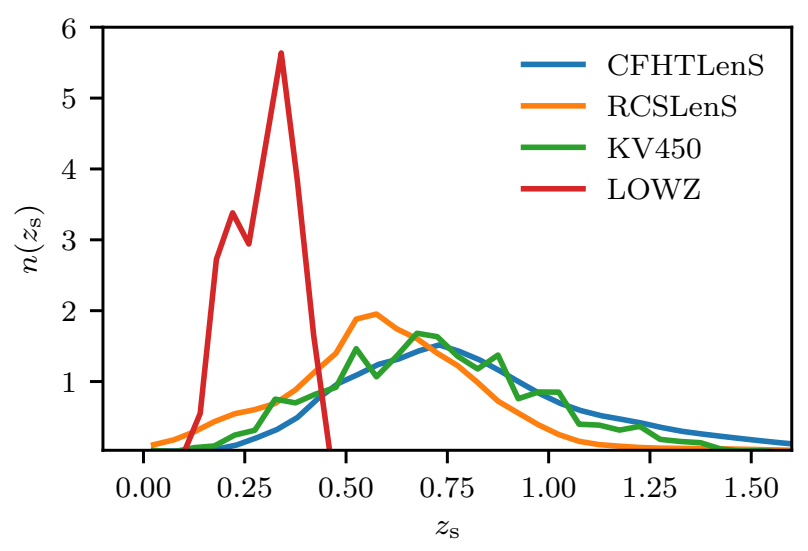

Fig. 1. The effective $n(z)$ of all three lensing surveys, as defined in Eq. (12), when using LOWZ galaxies as lenses. Each curve is normalised such that $\int n(z) \mathrm{d} z=1$.

\begin{tabular}{|c|c|c|c|}
\hline Survey & Patch name & $A_{\mathrm{eff}}^{\mathrm{LOWZ}}$ & $N_{\text {fil }}, 3 \sim 5 h^{-1} \mathrm{Mpc}$ \\
\hline \multirow[t]{3}{*}{ CFHTLenS } & $\mathrm{W} 1^{\triangle}$ & 53.38 & 1106 \\
\hline & $\mathrm{W}^{\triangle}{ }^{\triangle}$ & 40.12 & 835 \\
\hline & $\mathrm{W} 4^{\triangle}$ & 20.34 & 528 \\
\hline \multirow{3}{*}{ KV450 } & G9 & 11.10 & 305 \\
\hline & $\mathrm{G} 12^{\triangle}$ & 30.08 & 586 \\
\hline & $\mathrm{G} 15^{\wedge}$ & 94.73 & 2150 \\
\hline \multirow[t]{10}{*}{ RCSLenS } & $\mathrm{R}^{2047^{\triangle}}$ & 40.27 & 2111 \\
\hline & R0133 & 14.25 & 642 \\
\hline & $\mathrm{R} 1040^{\triangle}$ & 26.94 & 580 \\
\hline & R1303 & 4.00 & 119 \\
\hline & $\mathrm{R} 1514^{\triangle}$ & 32.72 & 1296 \\
\hline & R1613 & 9.16 & 331 \\
\hline & $\mathrm{R} 1645^{\triangle}$ & 22.66 & 678 \\
\hline & $\mathrm{R} 2143^{\triangle}$ & 42.16 & 1063 \\
\hline & $\mathrm{R} 2329^{\triangle}$ & 32.09 & 773 \\
\hline & R2338 & 0.39 & 25 \\
\hline
\end{tabular}

Table 2. Effective area and number of filaments in each survey patch.

Notes. The patches that are used in the analysis are identified with a $\triangle$.

their redshifts, position angles and number density in the patch. As we do not expect any physical signal from the random catalogue, we subtract any measured "random" signal from the data as follows:

$E_{\mathrm{f}}^{\mathrm{cor}}=E_{\mathrm{f}}-\overline{E_{\mathrm{ran}}}$.

Here, for a patch with $N_{\mathrm{p}}$ filament candidates $\overline{E_{\mathrm{ran}}}$ is given by

$\overline{E_{\mathrm{ran}}}=\frac{1}{N_{\mathrm{R}}} \frac{1}{N_{\mathrm{p}}} \sum_{r=1}^{N_{\mathrm{R}}} w_{\mathrm{ran}} \sum_{k=1}^{N_{\mathrm{p}}} E_{r}$,

and

$w_{\text {ran }}=\sum_{k=1}^{N_{\mathrm{p}}} w_{\mathrm{f}} W_{\mathrm{f}} / \sum_{k=1}^{N_{\mathrm{p}}} W_{r}$,

where $E_{r}$ and $W_{r}$ are the weighted shear (Eq. (6)) and weight (Eq. (8)) but measured around a random pair. $w_{\text {ran }}$ is the normalisation weight where $N_{R}$ is the number of realisations which 
ensures $N_{\mathrm{R}} \times N_{\mathrm{p}}$ exceeds 100000 in each patch ${ }^{4}$. This ensures that the random signal has low scatter so that we can take the mean as the random correction.

The random-corrected shear map $E_{\mathrm{f}}^{\text {cor }}$ and weight map $W_{\mathrm{f}}$ are then optimally combined over all filament candidates to determine the total weighted shear signal, $\mathcal{T}$, over the full sample,

$\mathcal{T}(i, j)=\frac{1}{K} \frac{\sum_{\mathrm{f}} w_{\mathrm{f}} E_{\mathrm{f}}^{\text {cor }}}{\sum_{\mathrm{f}} w_{\mathrm{f}} W_{\mathrm{f}}}$

where $w_{\mathrm{f}}=w_{\text {lens } 1} \times w_{\text {lens } 2}$ is the product of the recommended SDSS completeness weights for the LOWZ galaxies to account for any extra galaxy pairs in the case of fibre collision. We have also applied the multiplicative calibration correction at this stage which is given by Velander et al. (2014) as

$K(i, j)=\frac{\sum_{\mathrm{f}} w_{\mathrm{f}} \sum_{\mathrm{s}} w_{\mathrm{s}}\left[\overline{\sum_{\text {crit }}^{-1}}\left(z_{\mathrm{f}}\right)\right]^{2}\left(1+m_{\mathrm{s}}\right) \Theta_{\mathrm{s}}(i, j)}{\sum_{\mathrm{f}} w_{\mathrm{f}} W_{\mathrm{f}}}$.

The total weighted shear $\mathcal{T}(i, j)$ is a combination of both the shear contribution from the haloes surrounding the LOWZ LRGs and the contribution from any filament that connects them.

In order to isolate the filament we apply the "nulling" procedure described in Appendix A to get a final measurement of the shear contribution from the filament only, $\mathcal{F}(r)$, as a function of the distance, $r$, from the central filament axis,

$\mathcal{F}(r)=\frac{\sum_{i=i_{\min }}^{i_{\max }} \mathcal{N}[\mathcal{T}(i, r)]}{i_{\max }-i_{\min }+1}$.

Here $\mathcal{N}$ is the nulling operator given in Eq. (A.11) and the summation over pixels from an $i_{\min }$ to $i_{\max }$ runs along the filament from $-0.438 R_{\text {sep }}$ to $0.438 R_{\text {sep }}$. This value was found to minimise any residual contribution from the haloes positioned at $\pm 0.5 R_{\text {sep }}$, that remains after a nulling analysis of the SLICS simulation (see Sect. 4.1). Our nulling operator $\mathcal{N}$ combines the shear values measured at 8 different positions (including 4 positions from a reflection about the filament axis) which alternatingly rotate around the two haloes. In this way the isotropic contribution from the parent haloes sum to zero (i.e. "null") and any anisotropic contribution in-between the two haloes can be recovered. In Appendix A, we provide a detailed proof and compare our nulling approach to that adopted in $\mathrm{C} 16$. Through tests on a fiducial model, we show that the $\mathrm{C} 16$ nulling approach produces a biased result on large scales.

In this derivation we have carried both components of the shear with $\mathcal{F}=\mathcal{F}_{\gamma_{1}}+i \mathcal{F}_{\gamma_{2}}$. Given the Mead et al. (2010) filament model, where $\kappa=-\gamma_{1}$, we expect $\mathcal{F}_{\gamma_{2}}=0$. We will fit $\mathcal{F}_{\gamma_{1}}$ using the two-parameter model in Eq. (5) with the amplitude parameter replaced by $\mathcal{F}_{\mathrm{c}}$ which is equivalent to $\Sigma_{\text {crit }} \kappa_{\mathrm{c}}$ for a single lenssource pair.

\subsection{Error estimation from SLICS}

In order to estimate the error on the measured signal from observations, we use a large number of independent and representative lensing simulations from SLICS for each of the three

\footnotetext{
4 For patches with $A_{\text {eff }}<20 \mathrm{deg}^{2}$ we found that the sampling variance between the random catalogues was too large and we therefore do not use these patches in the final analysis.
}

surveys. SLICS allows us to correctly account for the sampling variance, which was found to be the dominant source of noise in Kondo et al. (2019). The source sample of galaxies differs for each filament pair owing to our source selection that $z_{\mathrm{B}}-z_{\mathrm{f}}>0.1$. For KV450 we can apply this source selection criteria accurately as the SLICS simulations include mock KV450 photometric redshifts that re-produce the scatter, bias and catastrophic outlier populations found in the KV450 data (Harnois-Déraps et al. 2018). For CFHTLenS and RCSLenS, this information is not encoded. We therefore create mocks from SLICS, modelling source samples for four different filament bins with $\left(z_{\min }, z_{\max }\right)=[0.1,0.2],[0.2,0.3],[0.3,0.4]$, and $[0.4,0.5]$, respectively. For each filament bin, we calculate the source galaxy redshift distribution $n(z)$, using Eq. (12) and the effective galaxy number density for sources with $z_{\mathrm{B}}-z_{\max }>0.1$. We then populate 500 independent simulations using these distributions, and measure and combine the weighted shear and weight maps for each of the four filament bins. For KV450 we are able to verify that this binned approach is consistent to the unbinned methodology applied to the data using the KV450 SLICS simulations.

The covariance matrix of the signal is reweighted by the number of filament candidates for each survey, $n_{\text {fil,survey }}$, and estimated from the SLICS simulations as

$$
\operatorname{Cov}=\frac{\bar{n}_{\text {fil sim }}}{n_{\text {fil,survey }}} \frac{1}{N_{\text {sim }}-1} \sum_{k=1}^{N_{\text {sim }}}\left(\mathcal{F}_{\gamma_{1}}^{k}-\overline{\mathcal{F}_{\gamma_{1}}}\right)\left(\mathcal{F}_{\gamma_{1}}^{k}-\overline{\mathcal{F}_{\gamma_{1}}}\right)^{T}
$$

where $\overline{\mathcal{F}_{\gamma_{1}}}$ is the filament signal (Eq. (18)) averaged over all $N_{\text {sim }}=500$ survey-specific SLICS simulations, and $\bar{n}_{\text {fil,sim }}$ is the average number of filament candidates in these simulations. The covariance is then used to calculate the $\chi^{2}$ when estimating parameters in the filament model as

$\chi_{\text {model }}^{2}=\left(\mathcal{F}_{\gamma_{1}}-\mathcal{F}_{\gamma_{1}}^{\mathrm{fit}}\right)^{T} \operatorname{Cov}^{-1}\left(\mathcal{F}_{\gamma_{1}}-\mathcal{F}_{\gamma_{1}}^{\mathrm{fit}}\right)$

where $\mathcal{F}_{\gamma_{1}}{ }^{\text {fit }}=\mathcal{F}_{\gamma_{1}}{ }^{\text {fit }}\left(\mathcal{F}_{\mathrm{c}}, r_{\mathrm{c}}, r\right)$ is our filament model defined in Eq. (5), calculated on a fine grid of parameters $\left(\mathcal{F}_{\mathrm{c}}, r_{\mathrm{c}}\right)$. In analogy to Eq. (19), we also define a covariance for the $\mathcal{F}_{\gamma_{2}}$ component which serves as a systematic null-test for our analysis.

We found that a simple bootstrap error analysis of the data, where the set of maps are resampled with repetitions before stacking, underestimates the true measurement error. This approach misses the sampling variance term which, like Kondo et al. (2019), we find is a significant component to the error for small-area surveys such as KV450 and CFHTLenS.

\subsection{Accounting for uncertainty in the redshift distributions}

As discussed in Sect. 2.1, the probability distribution $\overline{p_{\mathrm{s}}}\left(z_{\mathrm{s}}, z_{\mathrm{l}}\right)$ from Eq. (11) have not been calibrated for RCSLenS and CFHTLenS. A systematic uncertainty on the resulting $n\left(z_{\mathrm{s}}\right)$ is thus expected. In order to take this into account, we use a nuisance parameter $\delta z_{\mathrm{s}}=0.1$ for RCSLenS and $\delta z_{\mathrm{s}}=0.04$ for CFHTLenS that captures the $p\left(z_{\mathrm{s}}\right)$ uncertainty determined by Choi et al. (2016). For KV450 we use $\delta z_{\mathrm{s}}=0.025$ following Wright et al. (2019). We shift the $\overline{p_{\mathrm{s}}}\left(z_{\mathrm{s}}, z_{1}\right)$ by $\pm \delta z_{\mathrm{s}}$ in Eq. (10) to yield two new functions $\overline{\Sigma_{\text {crit }}^{-1}}\left(z_{1}\right)^{ \pm}$, and repeat the full measurement and error analysis using both the $\overline{\Sigma_{\text {crit }}^{-1}}\left(z_{1}\right)^{+}$and $\overline{\Sigma_{\text {crit }}^{-1}}\left(z_{1}\right)^{-}$ calibration. We then estimate the likelihood of the data $D$ given the model $\left(\mathcal{F}_{\mathrm{c}}, r_{\mathrm{c}}\right)$, assuming a prior on the uncertainty $\delta z_{\mathrm{s}}$ which 


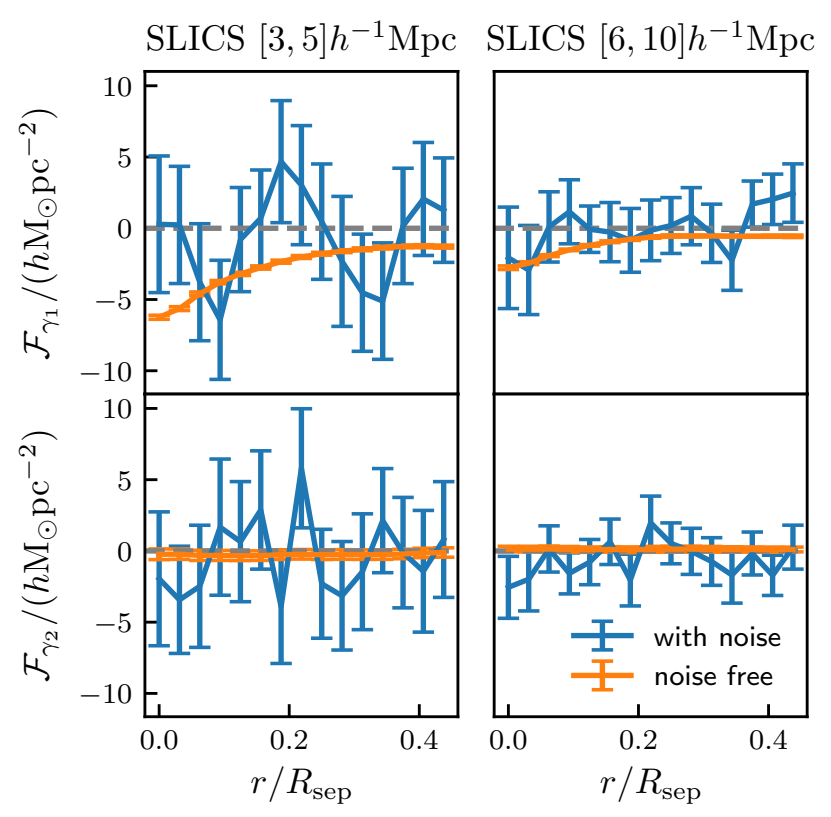

Fig. 2. Weighted shear measured between LRG pairs in the SLICS simulation of a random $400 \mathrm{deg}^{2}$ degree survey. Left: results from 3 to $5 h^{-1} \mathrm{Mpc}$ filament candidates. Upper panel: average surface mass density $\mathcal{F}_{\gamma_{1}}$, and the lower panel shows the measured cross-shear component. On both panels, the blue data shows the result for a KiDS-like survey depth and shape noise, and the orange data points show the measurement for a noise-free simulation with the errorbar given by the error on the mean of all 158 realisations. Right: set of results from 6 to $10 \mathrm{~h}^{-1} \mathrm{Mpc}$ filament candidates.

consists of $3 \delta$-functions and marginalised over as

$$
\begin{aligned}
\mathcal{L}\left(D \mid \mathcal{F}_{\mathrm{c}}, r_{\mathrm{c}}\right) & =\mathcal{L}\left(D \mid \mathcal{F}_{\mathrm{c}}, r_{\mathrm{c}},-\delta z_{\mathrm{s}}\right)+\mathcal{L}\left(D \mid \mathcal{F}_{\mathrm{c}}, r_{\mathrm{c}}, 0\right)+\mathcal{L}\left(D \mid \mathcal{F}_{\mathrm{c}}, r_{\mathrm{c}}, \delta z_{\mathrm{s}}\right) \\
& \propto \exp \left(-\frac{1}{2} \chi_{-\delta z_{\mathrm{s}}}^{2}\right)+\exp \left(-\frac{1}{2} \chi^{2}\right)+\exp \left(-\frac{1}{2} \chi_{\delta z_{\mathrm{s}}}^{2}\right)
\end{aligned}
$$

A full marginalisation where many samples are taken at different redshift offsets, spanning the $\delta z$ range, is unfortunately unfeasible given the complexity of the measurement pipeline.

\section{Results}

\subsection{Filaments in SLICS}

We validated our pipeline using the SLICS simulations of mock LOWZ lens galaxies and mock KiDS sources. We analysed both a noise-free catalogue and a catalogue with shape noise and mock photometric redshifts. The result is shown in Fig. 2 where the upper panels show measurements of $\mathcal{F}_{\gamma_{1}}$, and the lower panels show measurements of $\mathcal{F}_{\gamma_{2}}$. The left and right columns correspond to results from the $3-5 h^{-1} \mathrm{Mpc}$ and $6-10 h^{-1} \mathrm{Mpc}$ filament candidates, respectively.

For the noise-free simulations, we find that $\mathcal{F}_{\gamma_{2}}$ is consistent with zero for both the 3-5 $h^{-1} \mathrm{Mpc}$ and 6-10 $h^{-1} \mathrm{Mpc}$ length filaments. This demonstrates that our nulling procedure correctly removes the contribution from the LRG haloes in the analysis. However, for the simulations with shape noise, both the $\mathcal{F}_{\gamma_{1}}$ and $\mathcal{F}_{\gamma_{2}}$ measurements are consistent with zero, which suggests that even though KV450 is deeper than the KiDS-450 data simulated in SLICS, we should not expect a significant detection from $\mathrm{KiDS}$ alone. As the variance in the noise-free simulation reflects the level of sample variance, we also report that, by measuring the noise level from these two sets of simulations, the sample variance is comparable with the shape noise.

Constraining the parameters of Mead et al. (2010) model with the noise-free SLICS results we find $\mathcal{F}_{\mathrm{c}}^{3-5}=5.61 \pm$ $0.55 h M_{\odot} \mathrm{pc}^{-2}, r_{\mathrm{c}}^{3-5}=0.40 \pm 0.04 h^{-1} \mathrm{Mpc}, \mathcal{F}_{\mathrm{c}}^{6-10}=2.25 \pm$ $0.14 h M_{\odot} \mathrm{pc}^{-2}$, and $r_{\mathrm{c}}^{6-10}=1.12 \pm 0.08 h^{-1} \mathrm{Mpc}$. The $\chi_{3-5}^{2}=$ 16.33 and $\chi_{6-10}^{2}=15.42$ demonstrate that the model is a good fit to the data $(v=13$ degrees of freedom). We find that the surface mass density of the filament is a factor of 2.5 smaller for the $6-10 h^{-1} \mathrm{Mpc}$ filament and will therefore be more challenging to detect using gravitational lensing.

With the noise-free simulations we are able to analyse whether our signal depends on the redshift of the filament. We constrain the amplitude $\mathcal{F}_{\mathrm{c}}$ and the scale $r_{\mathrm{c}}$ parameters of the filament model for 4 redshift quantiles of the SLICS LOWZ filament samples using the same background sources, with the result shown in Fig. 3. The choice of parameterisation on the axes is motivated by the filament model equation as well as for visual simplicity. Here we see significant differences between the samples which could be caused by an evolution in the bias of the LOWZ-like galaxy samples in the SLICS mocks, or the evolution of the filament density field. Given the different source redshift distributions of the three lensing surveys (see Fig. 1) which makes the effective redshift of the average lens differ, this result suggests that we should not necessarily expect the results of these surveys to agree perfectly.

\subsection{The detection of filaments with KV450, RCSLenS and CFHTLenS}

Figure 4 presents our filament shear measurements and constraints on the two parameters of the filament model (Eq. (5)) for physical pairs, our filament candidates (upper panels), and non-physical pairs, our control sample (lower panels). The left panel shows the nulled $\mathcal{F}(r)$ shear signal as a function of the distance from the centre of the filament measured in units of $h M_{\odot} \mathrm{pc}^{-2}$. The result is presented for each lensing survey. We also show the measurements from the three-surveys combined using inverse variance weighting. The blue data points are a measurement of $\mathcal{F}_{\gamma_{1}}$, whereas the orange data points show the nulltest $\mathcal{F}_{\gamma_{2}}$. The shaded region corresponds to the statistical noise from our fiducial measurements, and the capped errorbars correspond to the systematic uncertainty captured by the photometric redshift bias nuisance parameter $\delta z$ (see Sect. 3.4). The right panel shows the $68 \%$ and $95 \%$ confidence region of parameters $\mathcal{F}_{\mathrm{c}}$ and $r_{\mathrm{c}}$ in Mead et al. (2010) filament model. These estimated parameters can be compared to the best-fit parameter from the noise-free SLICS analysis in Sect. 4.1 which is also represented by the cross in the right panel. We note that the noise-free SLICS best-fit is consistent with all three surveys. We also present joint constraints from the combined signal using a block covariance

$\operatorname{Cov}_{\text {all }}=\left(\begin{array}{ccc}\operatorname{Cov}_{\mathrm{CF}} & 0 & 0 \\ 0 & \operatorname{Cov}_{\mathrm{RC}} & 0 \\ 0 & 0 & \operatorname{Cov}_{\mathrm{KV}}\end{array}\right)$

and extended data vector $\mathcal{F}_{\text {ext }}=\left(\mathcal{F}_{\mathrm{CF}}, \mathcal{F}_{\mathrm{RC}}, \mathcal{F}_{\mathrm{KV}}\right)^{\mathrm{T}}$, providing an estimate of the average filament profile from all the filament candidates across the three surveys. Equation (22) assumes that the surveys are uncorrelated, which is a good approximation to make given the lack of overlap between the different surveys.

To quantify the significance of our measurements, we use the likelihood ratio test between the null hypothesis $H_{0}$ and the 

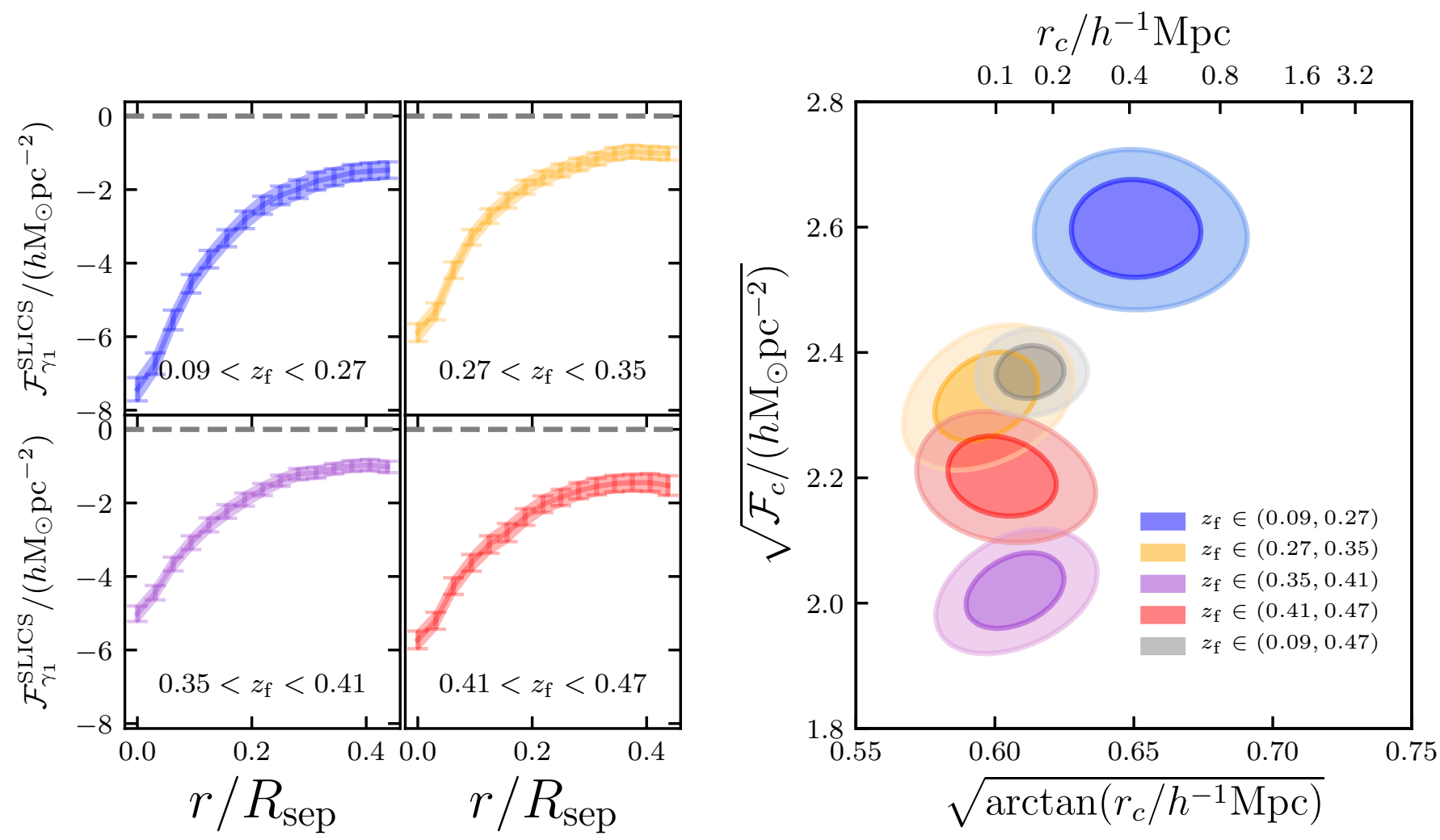

Fig. 3. Redshift evolution of the filament signal in the noise-free SLICS simulation. Left panel: $\mathcal{F}_{\gamma_{1}}$ signal measured from filament candidates in SLICS for four redshift quantiles. Right panel: $68 \%$ and $95 \%$ confidence region of the model parameters $\left(\mathcal{F}_{\mathrm{c}}, r_{\mathrm{c}}\right)$ from the corresponding signal in the left panel, with the result from all samples combined shown in grey.

filament model $H_{1}$, where the likelihood ratio LR is

$\operatorname{LR}(\mathcal{F}(r))=\frac{\sup _{\theta \in B_{1}} \mathcal{L}(\theta \mid \mathcal{F}(r))}{\sup _{\theta \in B_{0}} \mathcal{L}(\theta \mid \mathcal{F}(r))}$,

where $B_{0}$ and $B_{1}$ are the parameter space in each hypothesis, i.e., $B_{0}$ has no free parameters and $B_{1}=\left\{\mathcal{F}_{\mathrm{c}}, r_{\mathrm{c}}\right\}$. By Wilks' theorem (Wilks 1938; Williams 2001), the deviance defined as Dev $=2 \ln$ LR has an asymptotic chi-squared distribution with $\operatorname{dim}\left(B_{1}\right)-\operatorname{dim}\left(B_{0}\right)=2$ degrees of freedom when $H_{0}$ is true. Estimating the maximum likelihoods from $\chi_{\text {null }}^{2}$ and $\chi_{\text {model,min }}^{2}$ using Eq. (21) and computing the deviance, we report the significance level for each individual survey as well as the combined analysis in Table 3. The reported $\chi_{\text {min,model }}^{2}$ suggests our model is a reasonable fit to the data in all cases even for KV450, where $p\left(\chi^{2}<7.40 \mid v=13\right)=0.12$. We find the best-fit model parameters for $\mathcal{F}_{\gamma_{1}}$ from all three surveys combined as $\mathcal{F}_{\mathrm{c}}=10.5 \pm 2.9 h M_{\odot} \mathrm{pc}^{-2}$ and $r_{\mathrm{c}}=0.4_{-0.1}^{+0.2} h^{-1}$ Mpc. We note that, the majority of the detection derives from CFHTLenS alone with a $3.1 \sigma$ detection. Combining all three surveys we measure a $3.4 \sigma$ detection of the filament weak lensing signal. CFHTLenS is the most constraining survey as it combines both survey depth with significant SDSS overlap. KV450, with roughly half the source density of CFHTLenS, and RCSLenS, with roughly $20 \%$ of the source density, will only start to add significant constraining power with the inclusion of additional overlapping SDSS area.

Our control sample of "non-physical pairs" (NP) were selected to be pairs of lens galaxies with projected separations $3-5 h^{-1} \mathrm{Mpc}$, but distant in redshift space $(0.033<\Delta z<0.04)$.
These non-physical pairs would not be connected by a filament, hence providing an important validation of our nulling approach to isolate the filament signal. We find that the measured signal for $\mathcal{F}_{\gamma_{1,2}}$ is consistent with zero for all surveys and the combined survey as shown in Table 3 and the lower panel of Fig. 4.

For consistency with other analyses in the literature we also analysed 27880 filament pairs in LOWZ that have a physical separation of $6-10 h^{-1} \mathrm{Mpc}$. In contrast to other studies, we do not detect a significant signal for these larger-separation filaments in any of the surveys individually. In combination we find a weak signal at $1.6 \sigma$ significance, with $\mathcal{F}_{\mathrm{c}}=1.3 \pm 0.6$. The half-maximum radius of the density profile, $r_{\mathrm{c}}$, is, however, unconstrained.

\section{Conclusions and discussion}

In this paper, we present a $3.4 \sigma$ detection of filamentary structure connecting luminous red galaxies separated by $3-5 h^{-1} \mathrm{Mpc}$. Through a series of null tests we have verified the robustness of this result. Our work extends the analysis presented in $\mathrm{C} 16$ by improving the methodology to null the weak lensing signal from the LRGs in order to isolate the weak lensing distortions induced by the filamentary structure alone. We note that this nulling method cannot distinguish between a pair of spherical haloes joined by a cylinder of matter, or two elliptical haloes which extend towards each other. Higuchi et al. (2014) shows that there is no hard line between a filament and its corresponding halo, with haloes typically extending along the filament. As we find a strong nulled signal on scales much larger than the typical $\left(\sim 1 h^{-1} \mathrm{Mpc}\right)$ virial radius of the LRGs (Parejko et al. 2013), we would argue that it is unlikely to originate from two perfectly 


\section{$[3,5] h^{-1} \mathrm{Mpc}-$ Filaments}
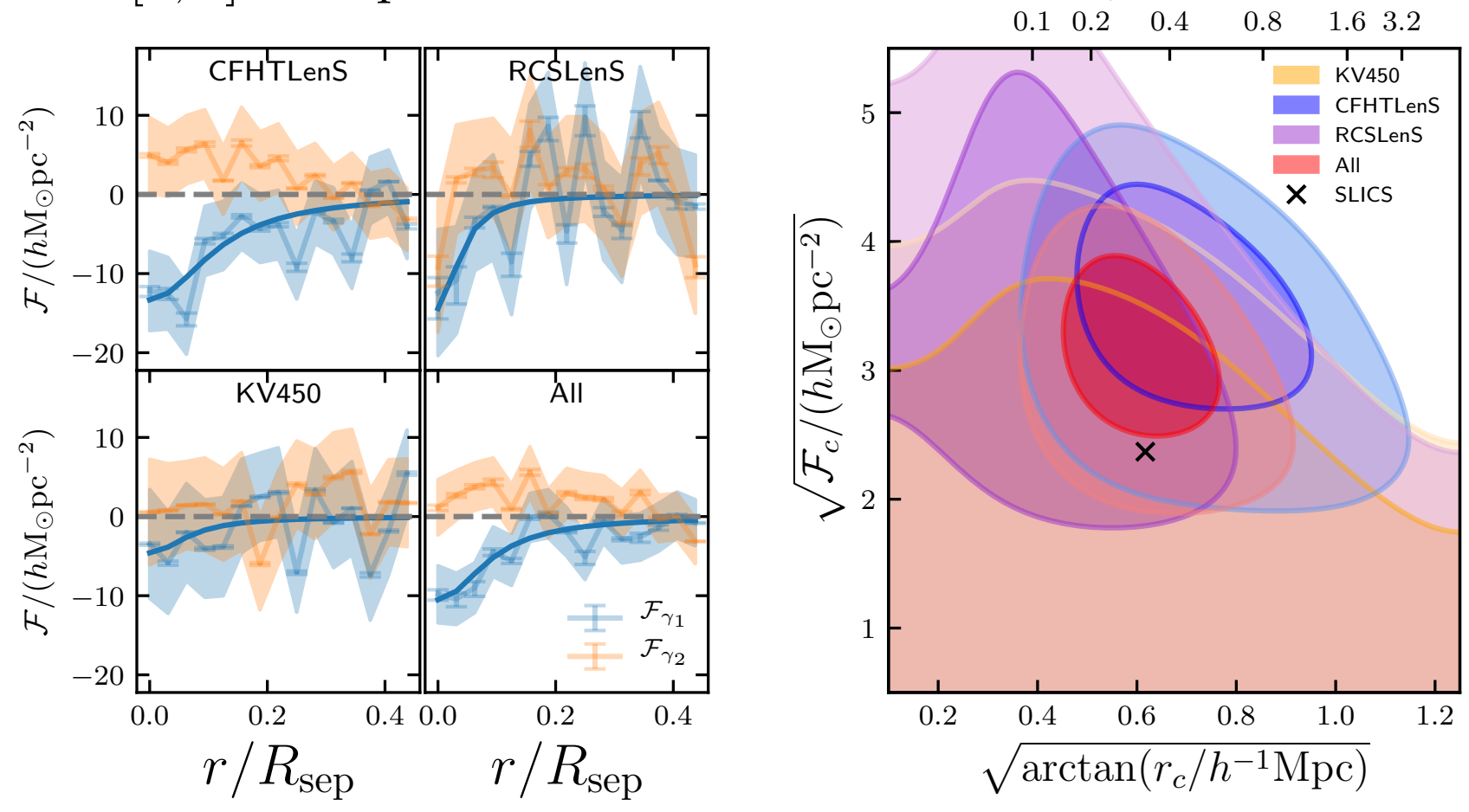

$[3,5] h^{-1} \mathrm{Mpc}-$ Non physicals

$$
r_{c} / h^{-1} \mathrm{Mpc}
$$
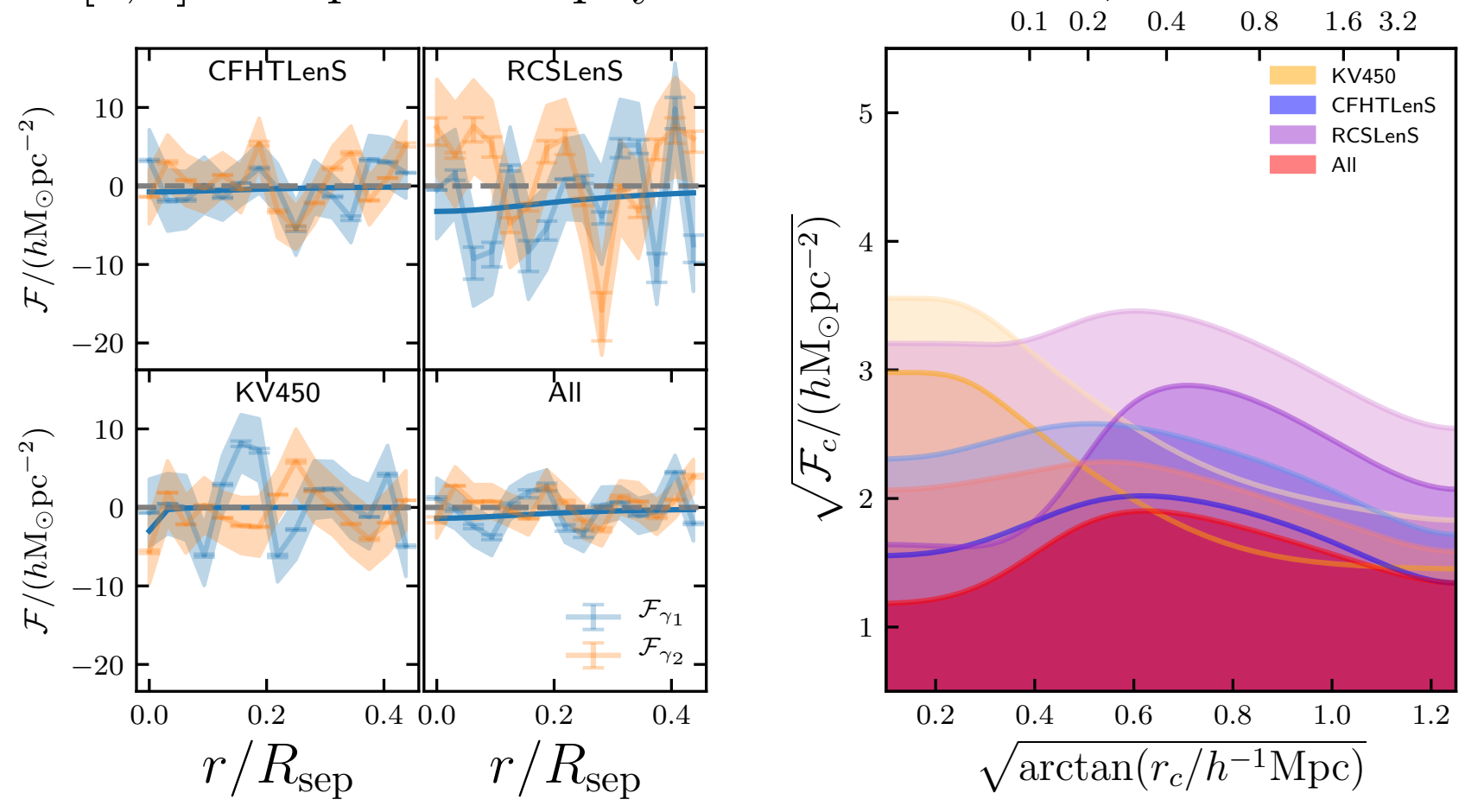

Fig. 4. The detection of the cosmic web between neighbouring luminous red galaxies as detected through the weak lensing of background galaxies from different lensing surveys. Left: $x$-axis is the distance measured perpendicular to the filament axis scaled such that 1 is equivalent to the projected separation between the pair of LRGs. The $y$-axis is the nulled shear signal where $\mathcal{F}_{\gamma_{1}}$ (blue data points) measures the average surface mass density of the filament, and $\mathcal{F}_{\gamma_{2}}$ is expected to be consistent with zero and hence serves as a null test. Lower right small panel: measurements from the three-surveys combined using inverse variance weighting. We note that this additional panel is purely for illustration, however, as our joint survey-constraints on the filament model are derived from a combination of the surveys on the likelihood-level. Right: estimated parameters in the filament density model Eq. (5) from the stacked signal for all surveys individually and their combination. Upper: results from $3 \sim 5 h^{-1} \mathrm{Mpc}$ Physical Pairs, our filament candidates; Lower: results from $3 \sim 5 h^{-1} \mathrm{Mpc}$ non-physical Pairs, our control sample. 
Table 3. $\chi^{2}$ value and $p$-value for all computed signals from each individual survey and their combination.

\begin{tabular}{|c|c|c|c|c|c|}
\hline & & CFHTLenS & RCSLenS & KV450 & All \\
\hline \multirow{7}{*}{ PP } & $\mathcal{F}_{\mathrm{c}} /\left(h M_{\odot} \mathrm{pc}^{-2}\right)$ & $13.3_{-4.0}^{+4.1}$ & $14.4_{-7.6}^{+8.5}$ & $4.6_{-4.5}^{+5.9}$ & $10.5_{-2.8}^{+2.9}$ \\
\hline & $r_{\mathrm{c}} /\left(h^{-1} \mathrm{Mpc}\right)$ & $0.5_{-0.2}^{+0.4}$ & $0.2_{-0.1}^{+0.3}$ & - & $0.4_{-0.1}^{+0.2}$ \\
\hline & $\chi_{\text {min,model }}^{2}$ & 12.2 & 11.6 & 7.5 & 40.4 \\
\hline & $\chi_{\min , \text { null }, \mathcal{F}_{\gamma_{1}}}^{2}$ & 24.6 & 15.6 & 8.1 & 55.0 \\
\hline & $\sigma_{\mathcal{F}_{\gamma_{1}}}$ & 3.08 & 1.49 & 0.35 & 3.40 \\
\hline & $\chi_{\text {min,null, }}^{2} \mathcal{F}_{\gamma_{2}}$ & 6.8 & 6.78 & 2.61 & 22.06 \\
\hline & $\sigma_{\mathcal{F}_{\gamma_{2}}}$ & 0.05 & 0.05 & $2 \mathrm{e}-4$ & $2 e-3$ \\
\hline \multirow{4}{*}{ NP } & $\chi_{\text {min,null, } \mathcal{F}_{\gamma_{1}}}^{2}$ & 9.2 & 20.0 & 19.3 & 53.8 \\
\hline & $\sigma_{\mathcal{F}_{\gamma_{1}}}$ & 0.17 & 1.38 & 1.28 & 1.36 \\
\hline & $\chi_{\min , \text { null }, \mathcal{F}_{\gamma_{2}}}^{2}$ & 12.3 & 20.7 & 5.8 & 43.0 \\
\hline & $\sigma_{\mathcal{F}_{\gamma_{2}}}$ & 0.44 & 1.45 & 0.02 & 0.59 \\
\hline
\end{tabular}

Notes. For each survey, PP means physical pair and NP stands for nonphysical pair. Both NP and $\mathcal{F}_{\gamma_{2}}$ serve as null tests for our analysis. For the combined signals, the degree of freedom is $v=45-2$. We also note that $r_{\mathrm{c}}$ is unconstrained by KV450.

aligned haloes (see Xia et al. 2017). But nevertheless we prefer to refer to our detection as that of filamentary structure, rather than that of a filament per se.

Previous studies have focused on LRGs separated by 6-10 $h^{-1} \mathrm{Mpc}$. Using all three lensing surveys (KV450, CFHTLenS and RCSLenS), we do not detect a significant signal for these larger-separation filaments in either the surveys individually, or in combination. This is in contrast to the significant $5 \sigma$ weak lensing detection of $6-10 h^{-1} \mathrm{Mpc}$ separation filaments reported by Epps \& Hudson (2017) using the same CFHTLenS dataset that has been analysed in this study. We report that we are unable to reproduce their result, even when adopting the same methodology. In comparison to $\mathrm{C} 16$, we find no comparable detection to their reported $\sim 4 \sigma$ detection. As we have shown that sampling variance makes a significant contribution to the overall error budget, the increase by a factor of 5 in the number of filaments studied by $\mathrm{C} 16$, in contrast to this analysis, is key to their detection, even though the C16 lensing source galaxy density is significantly shallower than the source densities of KV450, CFHTLenS and RCSLenS. Neither Epps \& Hudson (2017) nor C16 constrain the parameters of the Mead et al. (2010) filament model, but in the case of $\mathrm{C} 16$ we can compare the amplitudes of the measured signals. When adopting the $\mathrm{C} 16$ nulling approach, we find the two shear measurements to be fully consistent. The mean amplitude of our measurement is about four times larger than the amplitude reported in Kondo et al. (2019) (see discussion in Appendix A). Given our error budget, however, the results are consistent.

Authors de Graaff et al. (2019) calculate the average density $\bar{\kappa}$ between galaxy pairs separated by $6-14 h^{-1} \mathrm{Mpc}$ using a CMB lensing convergence map, finding $\rho_{0} \approx 5.5 \pm 2.9 \bar{\rho}(z)$. This estimate assumes that the matter density follows a cylindrical filament model, with density

$\rho\left(\ell, r_{\perp}\right)=\rho_{0} \exp \left(-\frac{r_{\perp}^{2}}{2 \sigma^{2}}\right) \exp \left(-\frac{\ell^{2}}{2 \sigma^{2}}\right)$,

where $\ell$ defines the size of the filament in the line-of-sight direction, $r_{\perp}$ defines the distance perpendicular to the filament axis on the projected sky and $\sigma$ is the intrinsic width. Integrating this density model over the line-of-sight, we can relate this to the surface mass density at the centre of the filament $\mathcal{F}_{\mathrm{c}}$ as

$\mathcal{F}_{\mathrm{c}}=\int \rho(\ell, 0) \mathrm{d} \ell=\sqrt{2 \pi} \sigma \rho_{0}$

For the value $\sigma=1.5 h^{-1} \mathrm{Mpc}$ adopted by de Graaff et al. (2019), and which is our best-fit amplitude parameter $\mathcal{F}_{\mathrm{c}}$, we find $\rho_{0}^{3-5}=(15.1 \pm 4.1) \bar{\rho}(z)$ for the $3-5 h^{-1} \mathrm{Mpc}$ filament sample at the average filament redshift $z=0.299$. For the $6-10 h^{-1} \mathrm{Mpc}$ filament sample we find $\rho_{0}^{6-10}=(1.9 \pm 0.9) \bar{\rho}(z)$, which is consistent with the de Graaff et al. (2019) result. Adapting the Mead et al. (2010) filament model, we can also integrate the model over the perpendicular distance and calculate the total mass enclosed between the two LRGs as

$M_{\text {fil }}\left(r_{\mathrm{c}}, \mathcal{F}_{\mathrm{c}}, R_{\text {sep }}\right)=R_{\text {sep }} \times 2 \int_{0}^{\infty} \mathcal{F}(r) \mathrm{d} r=\pi r_{\mathrm{c}} R_{\text {sep }} \mathcal{F}_{\mathrm{c}}$.

Taking the best-fit parameters $\mathcal{F}_{\mathrm{c}}$ and $r_{\mathrm{c}}$ for $3-5 h^{-1} \mathrm{Mpc}$ measurement, we find $M_{\text {fil }}=4.9 \pm 2.0 \times 10^{13} h^{-1} M_{\odot}$. It is worth noting that this estimate is based on the approximation that the deflection potential vanishes along the filament major axis. A more detailed analysis would attempt to obtain the excess mass map under the framework of galaxy-galaxy-galaxy lensing (Simon et al. 2008, 2019) but with a much larger separation of galaxy pairs.

Looking forward to upcoming deep weak lensing surveys, such as the European Space Agency's Euclid mission ${ }^{5}$ and the Large Synoptic Survey Telescope (LSST ${ }^{6}$ ), and deep spectroscopic surveys such as the Dark Energy Spectroscopic Instrument $\left(\mathrm{DESI}^{7}\right)$, the methodology that we present in this paper could be used to probe filamentary structure as a function of LRG mass and redshift. The combination of overlapping weak lensing surveys and spectroscopic surveys will provide the optimal datasets with which to fully explore the cosmic web.

Acknowledgements. We acknowledge support from the European Research Council under grant numbers 647112 (QX, CH, AA, MA, JHD, TT) and 770935 $(\mathrm{HH}, \mathrm{AW}) . \mathrm{CH}$ also acknowledges the support from the Max Planck Society and the Alexander von Humboldt Foundation in the framework of the Max PlanckHumboldt Research Award endowed by the Federal Ministry of Education and Research. YC acknowledges the support of the Royal Society through the award of a University Research Fellowship and an Enhancement Award. We acknowledge support from the European Commission under a Marie-Sklodwoska-Curie European Fellowship under project numbers 656869 (JHD) and 797794 (TT). $\mathrm{HH}$ is supported by a Heisenberg grant of the Deutsche Forschungsgemeinschaft (Hi 1495/5-1). AK acknowledges support from Vici grant 639.043.512, financed by the Netherlands Organisation for Scientific Research (NWO). PS acknowledges support from the Deutsche Forschungsgemeinschaft in the framework of the TR33 "The Dark Universe". Computations for the $N$-body simulations were performed in part on the Orcinus supercomputer at the WestGrid HPC consortium (www.westgrid.ca), in part on the GPC supercomputer at the SciNet HPC Consortium. SciNet is funded by: the Canada Foundation for Innovation under the auspices of Compute Canada; the Government of Ontario; Ontario Research Fund - Research Excellence; and the University of Toronto. Funding for SDSS-III has been provided by the Alfred P. Sloan Foundation, the Participating Institutions, the National Science Foundation, and the U.S. Department of Energy Office of Science. The SDSS-III web site is http://www. sdss3. org/. We thank SDSS-III for making their data products so easily accessible. SDSSIII is managed by the Astrophysical Research Consortium for the Participating Institutions of the SDSS-III Collaboration including the University of Arizona, the Brazilian Participation Group, Brookhaven National Laboratory, Carnegie Mellon University, University of Florida, the French Participation Group, the German Participation Group, Harvard University, the Instituto de Astrofisica de Canarias, the Michigan State/Notre Dame/JINA Participation Group, Johns Hopkins University, Lawrence Berkeley National Laboratory, Max Planck

\footnotetext{
5 http://www.euclid-ec.org

6 http://www. lsst.org

http://desi.lbl.gov/
} 
Institute for Astrophysics, Max Planck Institute for Extraterrestrial Physics, New Mexico State University, New York University, Ohio State University, Pennsylvania State University, University of Portsmouth, Princeton University, the Spanish Participation Group, University of Tokyo, University of Utah, Vanderbilt University, University of Virginia, University of Washington, and Yale University. Author contributions: All authors contributed to the development and writing of this paper. The authorship list is given in two groups: the lead authors (QX, NR, CH), followed by an alphabetical group who contributed to either the scientific analysis or the data products.

\section{References}

Alam, S., Albareti, F. D., Allende Prieto, C., et al. 2015, ApJS, 219, 12 Amon, A., Heymans, C., Klaes, D., et al. 2018, MNRAS, 477, 4285

Aragón-Calvo, M. A., van de Weygaert, R., \& Jones, B. J. T. 2010, MNRAS, 408, 2163

Bartelmann, M., \& Schneider, P. 2001, Phys. Rep., 340, 291

Benítez, N. 2000, ApJ, 536, 571

Bond, J. R., Kofman, L., \& Pogosyan, D. 1996, Nature, 380, 603

Bregman, J. N. 2007, ARA\&A, 45, 221

Briel, U. G., \& Henry, J. P. 1995, A\&A, 302, L9

Cheng, H., \& Gupta, K. C. 1989, ASME Trans. Ser. E: J. Appl. Mech., 56, 139

Choi, A., Heymans, C., Blake, C., et al. 2016, MNRAS, 463, 3737

Clampitt, J., Miyatake, H., Jain, B., \& Takada, M. 2016, MNRAS, 457, 2391

Colberg, J. M., Krughoff, K. S., \& Connolly, A. J. 2005, MNRAS, 359, 272

Colless, M., Dalton, G., Maddox, S., et al. 2001, MNRAS, 328, 1039

de Graaff, A., Cai, Y.-C., Heymans, C., \& Peacock, J. A. 2019, A\&A, 624, A48

Dietrich, J. P., Werner, N., Clowe, D., et al. 2012, Nature, 487, 202

Dolag, K., Meneghetti, M., Moscardini, L., Rasia, E., \& Bonaldi, A. 2006, MNRAS, 370, 656

Dvornik, A., Hoekstra, H., Kuijken, K., et al. 2018, MNRAS, 479, 1240

Eardley, E., Peacock, J. A., McNaught-Roberts, T., et al. 2015, MNRAS, 448, 3665

Ebeling, H., Barrett, E., \& Donovan, D. 2004, ApJ, 609, L49

Epps, S. D., \& Hudson, M. J. 2017, MNRAS, 468, 2605

Harnois-Déraps, J., \& van Waerbeke, L. 2015, MNRAS, 450, 2857
Harnois-Déraps, J., Amon, A., Choi, A., et al. 2018, MNRAS, 481, 1337

Heymans, C., Van Waerbeke, L., Bacon, D., et al. 2006, MNRAS, 368, 1323 Heymans, C., Van Waerbeke, L., Miller, L., et al. 2012, MNRAS, 427, 146 Higuchi, Y., Oguri, M., \& Shirasaki, M. 2014, MNRAS, 441, 745

Hildebrandt, H., Erben, T., Kuijken, K., et al. 2012, MNRAS, 421, 2355

Hildebrandt, H., Choi, A., Heymans, C., et al. 2016, MNRAS, 463, 635

Hildebrandt, H., Viola, M., Heymans, C., et al. 2017, MNRAS, 465, 1454

Hildebrandt, H., Köhlinger, F., van den Busch, J. L., et al. 2018, A\&A, accepted, https://doi .org/10.1051/0004-6361/201834878

Kondo, H., Miyatake, H., Shirasaki, M., Sugiyama, N., \& Nishizawa, A. J. 2019, ArXiv e-prints [arXiv: 1905.08991]

Kull, A., \& Böhringer, H. 1999, A\&A, 341, 23

Macciò, A. V., Dutton, A. A., van den Bosch, F. C., et al. 2007, MNRAS, 378, 55

Mandelbaum, R., Hirata, C. M., Seljak, U., et al. 2005, MNRAS, 361, 1287

Mead, J. M. G., King, L. J., \& McCarthy, I. G. 2010, MNRAS, 401, 2257

Miller, L., Heymans, C., Kitching, T. D., et al. 2013, MNRAS, 429, 2858

Parejko, J. K., Sunayama, T., Padmanabhan, N., et al. 2013, MNRAS, 429, 98

Pimbblet, K. A., \& Drinkwater, M. J. 2004, MNRAS, 347, 137

Ross, A. J., Percival, W. J., Sánchez, A. G., et al. 2012, MNRAS, 424, 564

Schneider, P., \& Watts, P. 2005, A\&A, 432, 783

Simon, P., Watts, P., Schneider, P., et al. 2008, A\&A, 479, 655

Simon, P., Saghiha, H., Hilbert, S., et al. 2019, A\&A, 622, A104

Springel, V., White, S. D. M., Jenkins, A., et al. 2005, Nature, 435, 629

Tanimura, H., Hinshaw, G., McCarthy, I. G., et al. 2019, MNRAS, 483, 223

Velander, M., Kuijken, K., \& Schrabback, T. 2011, MNRAS, 412, 2665

Velander, M., van Uitert, E., Hoekstra, H., et al. 2014, MNRAS, 437, 2111

Werner, N., Finoguenov, A., Kaastra, J. S., et al. 2008, A\&A, 482, L29

Wilks, S. S. 1938, Ann. Math. Stat., 9, 60

Williams, D. 2001, Weighing the Odds: A Course in Probability and Statistics (Cambridge: Cambridge University Press)

Wright, A. H., Hildebrandt, H., Kuijken, K., et al. 2019, A\&A, 632, A34

Wright, C. O., \& Brainerd, T. G. 2000, ApJ, 534, 34

Xia, Q., Kang, X., Wang, P., et al. 2017, ApJ, 848, 22

Zehavi, I., Zheng, Z., Weinberg, D. H., et al. 2011, ApJ, 736, 59

Zel'dovich, Y. B. 1970, A\&A, 5, 84 


\section{Appendix A: Remarks on the nulling technique}

In this appendix, we review the C16 nulling technique and develop an improved methodology to isolate the weak lensing signal from filaments. To explain the motivation behind nulling, we start with a single circularly symmetric halo positioned at the origin $(0,0)$, for which the complex shear is given by

$\gamma(\boldsymbol{r})=\gamma(r, \theta)=\gamma_{1}+\mathrm{i} \gamma_{2}=-(\bar{\kappa}-\kappa) \mathrm{e}^{2 \mathrm{i} \theta}$,

where $\bar{\kappa}$ is the mean convergence inside $r$. We define its counterpart $\gamma^{\mathrm{c}}$ as the complex shear at the same radial position, with a clockwise rotation of $90^{\circ}$, such that

$\gamma^{\mathrm{c}}(\boldsymbol{r}) \equiv \gamma\left(r, \theta+\frac{\pi}{2}\right)=-\gamma(r, \theta)$.

The counterpart is therefore able to "null" the shear, as $\gamma^{\mathrm{c}}(\boldsymbol{r})+$ $\gamma(\boldsymbol{r})=0$.

For a two-halo system as shown in Fig. A.1, the shear at each position $(r, \theta)$ is composed with the shear from halo $h_{1}$ and the shear from halo $h_{2}$. We can write this as

$\gamma(r, \theta)=\gamma_{\mathrm{h}_{1}}(t, \varphi)+\gamma_{\mathrm{h}_{2}}(s, \phi)$,

where the co-ordinates $(t, \varphi)$ are defined with halo $h_{1}$ at the origin, and the co-ordinates $(s, \phi)$ are defined with halo $h_{2}$ at the origin. Starting from position $P_{0}=\left(r_{0}, \theta_{0}\right)$, shear is given by

$P_{0}: \gamma\left(r_{0}, \theta_{0}\right)=\gamma_{\mathrm{h}_{1}}\left(t_{0}, \varphi_{0}\right)+\gamma_{\mathrm{h}_{2}}\left(s_{0}, \phi_{0}\right)$.

A clockwise rotation around halo $h_{1}$ by $90^{\circ}$ takes us to position $P_{1}$ at $\left(r_{1}, \theta_{1}\right)$. The shear here is given by

$P_{1}: \gamma\left(r_{1}, \theta_{1}\right)=\gamma_{\mathrm{h}_{1}}\left(t_{0}, \varphi_{0}+\frac{\pi}{2}\right)+\gamma_{\mathrm{h}_{2}}\left(s_{1}, \phi_{1}\right)$.

At position $P_{1}$, we see the shear contribution from halo $h_{1}$ is the counterpart to the shear contribution from halo $h_{1}$ at position $P_{0}$. We next rotate around halo $h_{2}$ by $90^{\circ}$ to position $P_{2}=\left(r_{2}, \theta_{2}\right)$, where the shear is given by

$P_{2}: \gamma\left(r_{2}, \theta_{2}\right)=\gamma_{\mathrm{h}_{1}}\left(t_{2}, \varphi_{2}\right)+\gamma_{\mathrm{h}_{2}}\left(s_{1}, \phi_{1}+\frac{\pi}{2}\right)$.

Similarly another $90^{\circ}$ rotation about halo $h_{1}$ (see Fig. A.2) to position $P_{3}$ gives

$P_{3}: \gamma\left(r_{3}, \theta_{3}\right)=\gamma_{\mathrm{h}_{1}}\left(t_{2}, \varphi_{2}+\frac{\pi}{2}\right)+\gamma_{\mathrm{h}_{2}}\left(s_{3}, \phi_{3}\right)$.

We note that, after another $90^{\circ}$ rotation about halo $h_{2}$, we come to position $P_{4}$ which is our starting point $P_{0}$. The sum of the shear from position $P_{0}, P_{1}, P_{2}$ and $P_{3}$ is given by

$$
\begin{aligned}
\sum_{i=0}^{3} \gamma\left(r_{i}, \theta_{i}\right) & =\gamma_{\mathrm{h}_{2}}\left(s_{0}, \phi_{0}\right)+\gamma_{\mathrm{h}_{2}}\left(s_{3}, \phi_{3}\right) \\
& =\gamma_{\mathrm{h}_{2}}\left(s_{3}, \phi_{3}+\frac{\pi}{2}\right)+\gamma_{h_{2}}\left(s_{3}, \phi_{3}\right)=0 .
\end{aligned}
$$

If we now add in a filament shear $\gamma_{\mathrm{f}}$ such that at each position $\gamma=\gamma_{\mathrm{f}}+\gamma_{h_{1}}+\gamma_{h_{2}}$, then

$\sum_{i=0}^{3} \gamma\left(r_{i}, \theta_{i}\right)=\gamma_{\mathrm{f}}\left(r_{0}, \theta_{0}\right)+\gamma_{\mathrm{f}}\left(r_{1}, \theta_{1}\right)+\gamma_{\mathrm{f}}\left(r_{2}, \theta_{2}\right)+\gamma_{\mathrm{f}}\left(r_{3}, \theta_{3}\right)$.

As we expect the filament shear profile to be symmetric about the horizontal axis, we also sum over the shear from positions

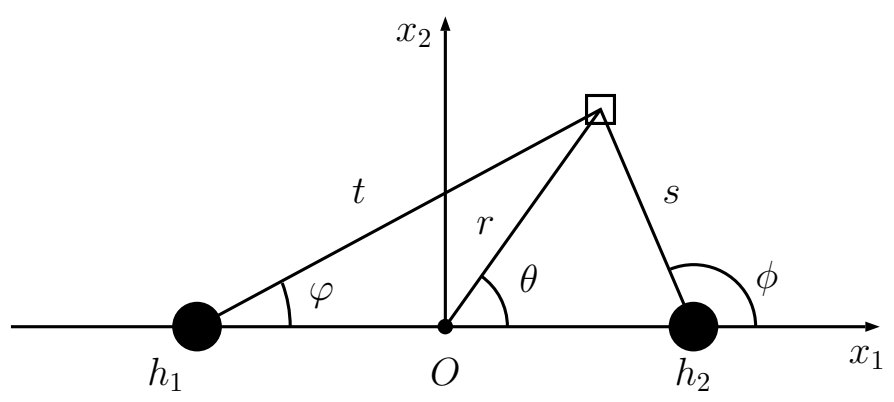

Fig. A.1. The bipolar configuration for two haloes centred symmetrically about origin $O$ at $h_{1}$ and $h_{2}$.

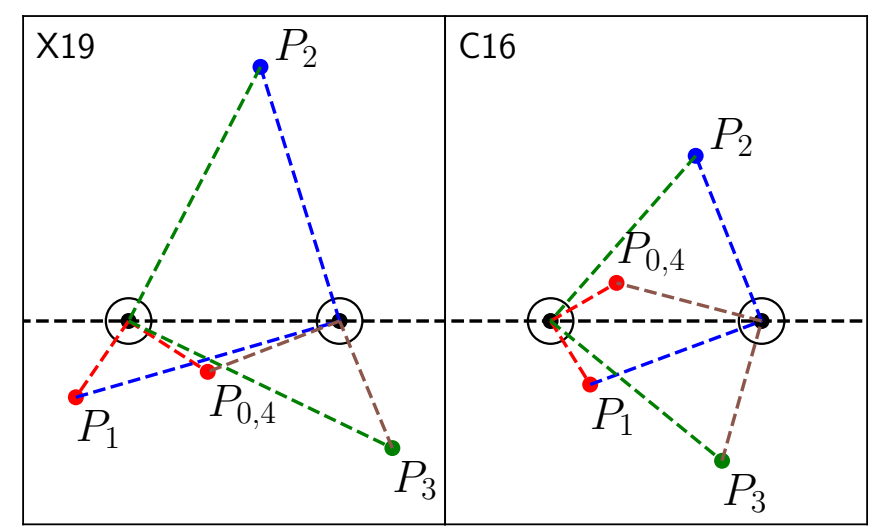

Fig. A.2. Illustration of the nulling technique. Every two line segments with the same colour represents an anti-clockwise rotation with respect to one halo. Right: configuration described in C16. Left: our adopted "X19" configuration which starts below the horizontal axis.

$P_{0}^{\prime}, P_{1}^{\prime}, P_{2}^{\prime}$ and $P_{3}^{\prime}$ that are reflections of $P_{0}, P_{1}, P_{2}$ and $P_{3}$ about the horizontal axis respectively, in order to get the average shear value at any distance away from filament axis. We therefore define the nulling operator $\mathcal{N}$ as

$\mathcal{N}\left[\gamma\left(r_{0}, \theta_{0}\right)\right]=\frac{1}{2}\left(\sum_{i=0}^{3} \gamma\left(r_{i}, \theta_{i}\right)+\sum_{i=0}^{3} \gamma\left(r_{i}^{\prime}, \theta_{i}^{\prime}\right)\right)$.

It is interesting to note that the above equations also apply when two halos are of different masses given their circular symmetry.

In Fig. A.2 we show two configurations for nulling. C16 chose to start $P_{0}$ above the horizontal axis and our work starts $P_{0}$ below the horizontal axis. We investigate the difference between these two approaches by first constructing a noiseless shear map from two NFW halo profiles using Eq. (14) in Wright \& Brainerd (2000) with $M_{\text {vir }}=10^{13} h^{-1} M_{\odot}$ and assuming a mass-concentration relation from Macciò et al. (2007). We assume both haloes are located at $z=0.3$ with background sources at redshift 0.7 , close to the mean value of KiDS. The resultant $\gamma_{1}$ map is shown in the top-left panel of Fig. A.3.

For each pixel $\left(x_{1}, x_{2}\right)$ on the map, we calculate $\mathcal{N}\left[\gamma_{i}\left(x_{1}, x_{2}\right)\right]$, and show in the top-right panel in Fig. A.3, the average of the sum along the horizontal axis, i.e., $\gamma_{1}^{\text {null }}(r)=$ $\sum_{x=x_{\max }}^{x_{\max }} \mathcal{N}\left[\gamma_{1}\left(x_{1}, x_{2}\right)\right] /\left(x_{\max }-x_{\min }+1\right)$. In the lower-right panel in Fig. A.3, we show the resulting $\mathcal{N}\left[\gamma_{2}\left(x_{1}, x_{2}\right)\right]$. As expected, the sum of nulling pixels are zeros (note the $10^{-16} / 10^{-18}$ on the $y$-axis). In the middle panel we repeat the analysis with the inclusion of a fiducial filament modelled using the power-law profile model in Eq. (5). The filament contribution to the shear $\gamma_{1}^{\mathrm{fil}}(r)$ is 

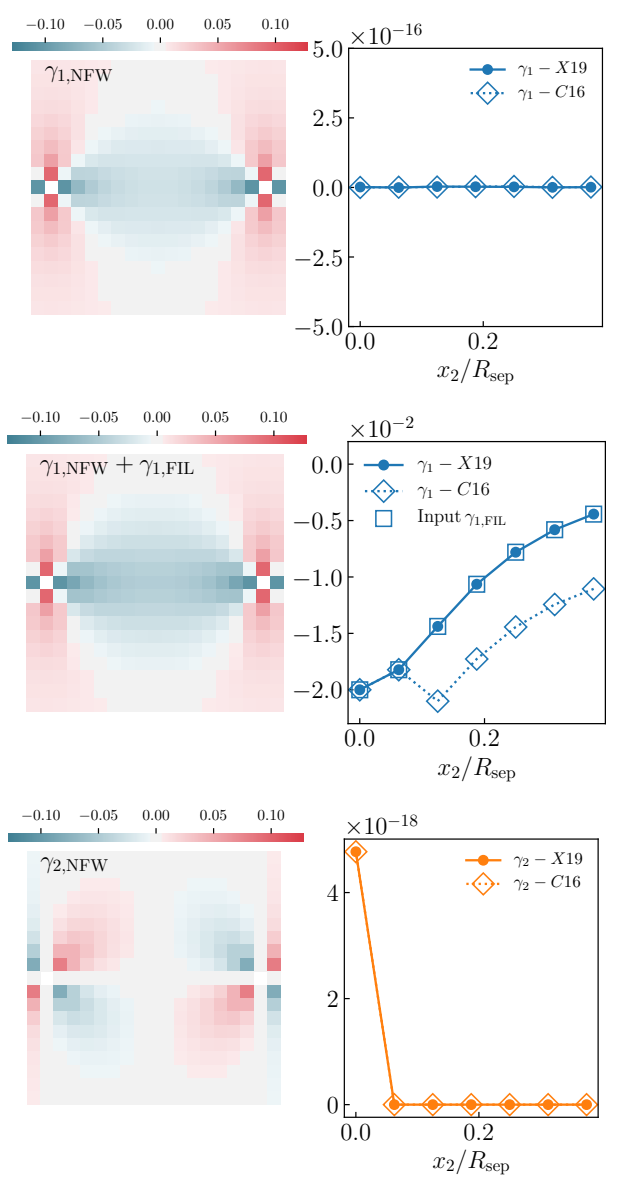

Fig. A.3. Left panel: shear maps from two NFW halos with or without fiducial filaments. Top row: $\gamma_{1}$ map generated by two NFW haloes only, while the middle row shows the $\gamma_{1}$ map with the addition of a fiducial filament profile. Lowest row: $\gamma_{2}$ map generated by two NFW haloes. it Right panel: results of the nulling procedure corresponding to the shear map on the left. In the top row, we see under both the X19 configuration (solid line) and C16's (dashed line), the resulting signal is consistent with zero. When adding a fiducial filament profile, in the middle row, we see our X19 configuration correctly recovers the input value whereas the $\mathrm{C} 16$ configuration is biased on large scales. In the lower row, we verify that both configurations null the $\gamma_{2}$ signal from two NFW haloes.

included in the 2D shear map as a power-law, symmetric about the $x_{1}$-axis (at $x_{2}=0$ ) with $k_{\mathrm{c}}=0.02$ and $r_{\mathrm{c}}=0.4 h^{-1} \mathrm{Mpc}$. These parameters were chosen to roughly replicate previous studies (Dolag et al. 2006), in order to model the true signal contrast in observations. If the nulling method is precise, we would expect the nulled filament signal measured from the map, $\gamma_{1}^{\text {null }}(r)$ to recover the input shear from the filament, $\gamma_{1}^{\text {fil }}(r)$, irrespective of the nulling method used. The middle-right panel shows that this is indeed the case, with our nulling method. The nulling method in $\mathrm{C} 16$, however, recovers a lower expectation value for the shear induced by the filament. This difference results from the C16 configuration. As shown in Eq. (A.10), for the C16 configuration, since $P_{0}$ and $P_{1}$ are both in the filament region, the nulling operator effectively mixes scales. This has an effect in producing a significant signal on large scales which does not reflect the underlying filament density profile. As $P_{1}, P_{2}$ and $P_{3}$ in our adopted configuration lies outside the bridge between two haloes, $\gamma_{\mathrm{f}}\left(r_{1}, \theta_{1}\right)+\gamma_{\mathrm{f}}\left(r_{2}, \theta_{2}\right)+\gamma_{\mathrm{f}}\left(r_{3}, \theta_{3}\right)$ is negligible. This enables us to recover the density profile accurately. We note that Kondo et al. (2019) adopted the C16 estimator but in their Eq. (9) (the equivalent of our Eq. (A.11)), they included an additional factor of 4 in the denominator, which, in our test-case in Fig. A.3 would result in the underestimation of the filament signal by a factor of 4 .

\section{Appendix B: Remarks on the spherical rotation}

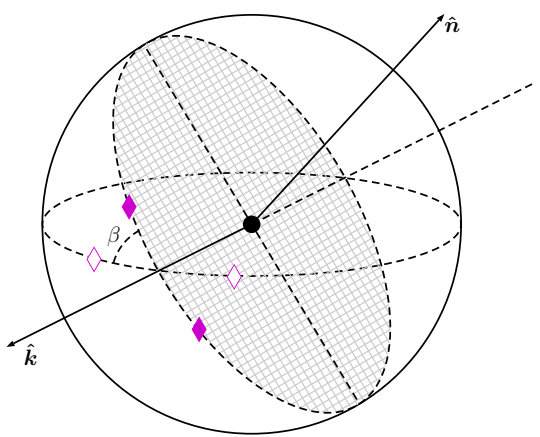

Fig. B.1. Illustration of the spherical rotation for filament pairs (solid pink diamonds). Open symbols are the rotated positions.

Here we detail the spherical rotation used in Sect. 3.2 to project all filaments onto the same reference frame. As illustrated in Fig. B.1, we rotate all galaxies about a given axis so that the filament (the shorter arc connected by solid pink diamonds) is transformed to lie horizontally on the equator (hollow pink diamonds). To do this, we first transfer the right ascension and declination onto a $3 \mathrm{D}$ vector on a unit sphere, such that their positions are $\boldsymbol{g}_{1}$ and $\boldsymbol{g}_{2}$. The normal vector is defined as $\hat{\boldsymbol{n}}=\boldsymbol{g}_{1} \times \boldsymbol{g}_{2}$. Noting the rotation axis lies on the equator and is perpendicular to the normal vector, we can write down the rotation axis $\hat{\boldsymbol{k}}$ and angle $\beta$ using components of $\hat{\boldsymbol{n}}$, so that

$\hat{\boldsymbol{k}}=\frac{\left(n_{2},-n_{1}, 0\right)^{\mathrm{T}}}{\sqrt{n_{1}^{2}+n_{2}^{2}}}$,
$\beta=\arccos \left(n_{3}\right)$.

We note that by defining $\boldsymbol{g}_{1}$ to be always on the left of $\boldsymbol{g}_{2}$, there is no ambiguity in the definition of the rotation axis, and the rotation angle will always lie between $\left[0, \frac{\pi}{2}\right]$. Rodrigues' rotation formula (Cheng \& Gupta 1989) then allows us to rotate every point on the sky to the desired frame where the filament pair now lies on the equator, such that for each source galaxy at position $\boldsymbol{g}_{\mathrm{s}}$, the new position is at:

$\boldsymbol{g}_{\text {new }}=\boldsymbol{g}_{\mathrm{s}} \cos \beta+\sin \beta\left(\hat{\boldsymbol{k}} \times \boldsymbol{g}_{\mathrm{s}}\right)+\left(\hat{\boldsymbol{k}} \cdot \boldsymbol{g}_{\mathrm{s}}\right)(1-\cos \beta) \hat{\boldsymbol{k}}$.

It is worth noting that because the shear was measured in each galaxy's local (RA, Dec) coordinate frame, the angle of rotation is different for different source galaxies. The transformed shear map $\left(\widetilde{e_{1}}, \widetilde{e_{2}}\right)$ for each galaxy is thus given by

$\left(\begin{array}{c}\widetilde{e_{1}} \\ \widetilde{e_{2}}\end{array}\right)=\left(\begin{array}{cc}\cos 2 \phi_{\mathrm{s}} & \sin 2 \phi_{\mathrm{s}} \\ -\sin 2 \phi_{\mathrm{s}} & \cos 2 \phi_{\mathrm{s}}\end{array}\right)\left(\begin{array}{l}e_{1} \\ e_{2}\end{array}\right)$,

where for each source galaxy, the rotation angle $\phi_{\mathrm{s}}$ is defined by the angular change of its local coordinate frame, for example, the declination to the $y$-axis of the filament. 